\title{
3-Fenil-5-(4-pridil)-1,2,4-triazol Molekülünün DFT(B3LYP/B3PW91)/HF ile Spektroskopik, Geometrik,Termodinamik ve Elektronik Analizi
}

\author{
Gül Kotan ${ }^{1 *}$, Haydar Yüksek ${ }^{2}$ \\ ${ }^{1}$ Kafkas Üniversitesi, KMYO, Kimya ve Kimyasal İşleme Teknolojileri Bölümü, Kars, Türkiye (ORCID: 0000-0002-4507-9029) \\ ${ }^{2}$ Kafkas Üniversitesi, Fen Edebiyat Fakültesi, Kimya Bölümü, Kars, Türkiye (ORCID: 0000-0003-1289-1800)
}

(İlk Geliş Tarihi 5 Kasım 2019 ve Kabul Tarihi 13 Aralık 2019)

(DOI: 10.31590/ejosat.643080)

ATIF/REFERENCE: Kotan, G. \& Yüksek, H. (2019). 3-Fenil-5-(4-pridil)-1,2,4-triazol Molekülünün DFT(B3LYP/B3PW91)/HF ile Spektroskopik, Geometrik,Termodinamik ve Elektronik Analizi. Avrupa Bilim ve Teknoloji Dergisi, (17), 933-943.

$\ddot{\mathbf{O} z}$

3-Fenil-5-(4-pridil)-1,2,4-triazol bileşiğinin moleküler geometrik optimizasyonu 6-311G(d,p) ve 3-21G temel setinde DFT(B3LYP, B3PW91)/ HF metodları kullanılarak elde edilmiştir. Böylece, bileşiğin en kararlı geometrik şekli çeşitli metod ve setlerle bulunmuştur. Deneysel spektral incelenmeler FT-IR değerleri ve ${ }^{1} \mathrm{H} /{ }^{13} \mathrm{C}-\mathrm{NMR}$ kimyasal kaymaları kullanılarak gerçekleştirilmiştir. Geometrik yapı analizleri (bağ açıları, bağ uzunlukları), Proton/ Karbon NMR kimyasal kaymaları, termodinamik parametreler, HOMO-LUMO analizleri, elektronik özellikler, mulliken yükleri, titreşimsel dalgalanmalar, dipol momenti, başlica molekülünün toplam enerjisi "Gaussian 09W" paket programı kullanılarak hesaplanmıştır. Sonuçlar, GaussView5.0 programında değerlendirilmiştir. Hesaplamalı IR verileri Veda4f programında belirlenmiş ve bu hormonik titreşim frekansları belirli faktörler ile ölçeklendirilmiştir. Deneysel değerler literatürden alınmıştır. Deneysel spektral değerler hesaplanan IR verileri ile karşılaştırılmıştır. Proton Nükleer Manyetik Rezonans ( $\left.{ }^{1} \mathrm{H}-\mathrm{NMR}\right)$ ve Karbon-13 Nükleer Manyetik Rezonans $\left({ }^{13} \mathrm{C}-\mathrm{NMR}\right)$ spektral değerleri, gaz fazında ve DMSO çözücüsünde GIAO yöntemine göre hesaplanmıştır. Bu değerler deneysel proton / karbon nmr verileriyle karşılaştırılmış ve sonuçlara göre regresyon analizi yapılmıştır. Ayrıca farklı yöntemlerle elde edilen ve fonksiyonel olan teorik sonuçlar birbiriyle karşılaştırılmıştır. Bundan başka, HOMO-LUMO enerji hesaplamaları ile kimyasal sertlik $(\eta)$, elektronegatiflik $(\chi)$, iyonlaşma potansiyeli (I), kimyasal yumuşaklık ( $\sigma$ ), elektron ilgisi (A) gibi parametreler belirlenmiştir. Elektron yoğunluğu moleküler yüzeyler, elektron spin potansiyeli (ESP), moleküler elektrostatik potansiyel (MEP), toplam yoğunluk ve kontur haritaları belirlenmiştir. Böylece farklı metodlar, fonksiyonlar ve setler ile molekülün tüm teorik parametreleri hesaplanmıştır. Sonuçta bu veriler karşılaştırılmış ve en uygun yöntem ve set bulunmuştur.

\section{Spektroscopic, Geometric, Termodynamic and Electronic Analysis with DFT(B3LYP/B3PW91)/HF of 3-Phenyl-5-(4-pyridyl)-1,2,4-triazole Molecule}

\begin{abstract}
The molecular geometric optimization of 3-Phenyl-5-(4-pyridyl)-1,2,4-triazole compound was obtained using DFT(B3LYP, B3PW91)/ HF methods at the 6-311G(d,p) and 3-21G basis sets. Thus, the most stable geometric conformer of the compound was found by various methods and sets. The experimental spectral investigations were performed using FT-IR values and ${ }^{1} \mathrm{H} /{ }^{13} \mathrm{C}-\mathrm{NMR}$ chemical shifts. Geometric structure analyses (bond angles, bond lengths), Proton/Carbon NMR chemical shifts, thermodynamic parameters, HOMO-LUMO analyses, electronic properties, mulliken charges, vibrational wavenumbers, dipole moment, total energy
\end{abstract}

\footnotetext{
${ }^{*}$ Sorumlu Yazar: Kafkas Üniversitesi, KMYO, Kimya ve Kimyasal İşleme Teknolojileri Bölümü, Kars, Türkiye ORCID: 0000-0002-4507-9029, gulkemer@hotmail.com
} 
of the title molecule were calculated using Gaussian $09 \mathrm{~W}$ software package. The results were improved at the GaussView5.0 program.

Computation IR data was determined at the Veda4f program and these harmonic vibrational frequencies were scaled with definite factor. Experimental data obtained from the literature. The experimental spectral values were compared with computed IR data. Proton Nuclear Magnetic Resonance ( $\left.{ }^{1} \mathrm{H}-\mathrm{NMR}\right)$ and Carbon-13 Nuclear magnetic Resonance $\left({ }^{13} \mathrm{C}-\mathrm{NMR}\right)$ spectral values was calculated in gas phase and in DMSO solvent according to GIAO method. These values were compared with experimental proton/ carbon NMR data and regression analysis were performed according to the results. Also, the theoretical results obtained with different methods and functional were compared with each other. Furthermore, parameters such as chemical hardness $(\eta)$, electronegativity $(\chi)$, ionization potential (I), chemical softness $(\sigma)$, electron affinity (A) were determined with HOMO-LUMO energies calculations. The molecular surfaces such as the electron density, the electron spin potential (ESP), molecular electrostatic potential (MEP), the total density and contour maps were designated. Thus, all theoretical parameters of molecule were calculated with different methods, functionals and sets. In the result, these data were compared and the most appropriate method and set were found.

Keywords: 1,2,4-Triazole, B3LYP, B3PW91, HOMO-LUMO, GIAO.

\section{Giriş}

3,5-Disübstitüe-1,2,4-triazol bileşiklerin sentezi için genel metod, alkilhidrazinler ile imin esterlerin kondenzasyonudur (Postovskii \& Vereshchagina, 1954; Brown \& Polya, 1962) Aril sübstütientlerini taşıyan 1,2,4-triazol türevleri, alkil gruplarını taşıyanlara nazaran daha kararlıdırlar ve reaksiyona girme yatkınlıkları daha fazladır. 1,2,4-Triazol içeren Schiff bazları ilaç ve koordinasyon kimyası için önemli bileşiklerdir ve antibiyotik, antitümör, antipsikolojik, antiallerjik gibi bazı önemli ilaçların sentezinde başlatıcı madde olarak kullanılmışlardır (Barton \& Ollis, 1979; Ingold, 1969; Layer, 1963). Ayrıca, günümüzde bilinen antifungal ajanların (Patel \& Park, 2014) yapısında 1,2,4-triazol taşıyan moleküller vardır bu yapılar tarımsal mantarların tedavisinde etkendir ve bu amaç için 1,2,4triazol türevleri birçok sebze ve mahsülün üretimini arttırmak için kullanılır (Fletcher ve ark., 2010). Ek olarak, antibakteriyel (Mari ve ark., 2008) antikanser (Krzysztof ve ark., 2008) antiinflammator (Tozkoparan ve ark., 2007), antioksidan (Yüksek ve ark., 2011) aktivite de gösterirler ve anastrozole (Buzdar, 2002; Wellington \& Faulds, 2002) estazolam (Vogel \& Morris, 1992; Cohn ve ark., 1992) ribavirin (Hong \& Cameron, 2002; Rockstroh, 2002; Graci, 2002) triazolam (Kanamitsu ve ark., 2000; Lobo \& Greene, 1997) gibi önemli ilaçların yapılarında 1,2,4-triazol halkası bulunmaktadır. Bundan başka boya ve plastik endüstride yaygın olarak kullanılmaktadırlar (Casaszar ve ark., 1985; Sheikhshoaie \& Sharif, 2006). Bu yapısal ve biyolojik özelliklerinden dolayı 1,2,4triazol türevlerinin dizaynı ve sentezi giderek artmıştır. 1,2,4-Triazol bileşiklerini içeren birçok çalışma mevcuttur. Bu bileşiklerin metal kompleksleri, eczacılık, tıp ve biyolojik sistemlerde yaygın bir şekilde kullanılmışırı ve oksidasyon, epoksidasyon ve hidroksilasyon reaksiyonlarında yüksek katalitik aktivite sergilemişlerdir (Prakash \& Adhikari, 2011; Gupta \& Sutar, 2008; Jia \& Li, 2014; Himeda ve ark., 2003; Cozzi, 2004).

Son yıllarda, hesaplamalı kimyanın gelişmesi ve moleküler modellemeye duyulan ihtiyacın artmasıyla teorik hesaplama metotları yaygın bir şekilde kullanılmaktadır. 1,2,4-Triazol bileşiklerinin de teorik tüm parametreleri incelenmiştir (Pokharia ve ark., 2017; Yüksek ve ark., 2017; Kotan \& Yüksek, 2016; Karunakaran \& Balachandran, 2012; Suvitha ve ark., 2014). Bunun için Kuantum kimyası hesaplamalarının temelini olușturan Yoğunluk Fonksiyonel Teorisi (DFT) ve Hatree Fock (HF) yöntemlerinden yararlanılmıştır (Frisch ve ark., 2009; Wolinski ve ark., 1990). Üç parametreli değiş tokuş-korelasyon fonksiyonu olan Perdew ve Wang (PW91) ve Lee-Yang-Parr korelasyon fonksiyonu (B3LYP ), elektron yoğunluğunu hesaplamayı hedefleyen DFT metodunun fonksiyonlarıdır (Becke, 1988; Becke, 1993; Lee ve ark., 1988; Perdew, 1986a; Perdew, 1992). Daha detaylı ve güvenilir sonuçlar sağlayan 6-311G(d,p) ve daha kısa sürede sonuçlanan 3-21G gibi temel setler ile hesaplama yapılmaktadır. Seçilen yöntemler ve setler ile bir molekülün yapısal özelliklerini, geometrisini, iç enerjisini, spektroskopik özelliklerini, elektronik ve termodinamik özelliklerini belirlemek mümkündür. ${ }^{13} \mathrm{C} /{ }^{1} \mathrm{H}-\mathrm{NMR}$ ve IR spektral verileri (Wolinski ve ark., 1990) teorik olarak aynı yöntem ve metodlar ile hesaplanarak, literatürden (Bahçeci ve ark., 2005) alınan deneysel spektral veriler ile karşılaştırılmıştır.

\section{Materyal ve Metot}

\subsection{Deneysel}

\section{3-Fenil-5-(4-pridil)-1,2,4-triazol Deneysel Spektral Verileri}

Reaksiyon sicaklığı: $200-205{ }^{\circ} \mathrm{C}$, Verim: $2.00 \mathrm{~g}(\% 90)$, E.n: $236-37{ }^{\circ} \mathrm{C}$; IR $\left(\mathrm{v}, \mathrm{cm}^{-1}\right): 3180(\mathrm{NH}), 1615-1595(\mathrm{C}=\mathrm{N}), 755,695$ (monosübstitüe benzen); ${ }^{1} \mathrm{H}-\mathrm{NMR}$ (400 MHz, DMSO-d 6 ): $\delta: 7.35-8.50$ (m, 9H, Ar-H), $14.30(\mathrm{~s}, 1 \mathrm{H}, \mathrm{NH}) ;{ }^{13} \mathrm{C}-\mathrm{NMR}(100 \mathrm{MHz}$, DMSO- $\mathrm{d}_{6}$ ): $\delta: 121.60,127.52,127.98,129.40,129.77,130.68,133.60,135.00,140.90,151.92,154.70$ (Ar-C), 158.20, 160.71 (Triazol-C).

\subsection{Hesaplama Detayları}

Sentezi gerçekleştirilmiş bir molekülün deneysel verilerini destekler nitelikte olan bu çalışma da, tüm hesaplamalar çok sayıda metod ve temel set seçeneğine sahip olan Gaussian 09W paket programı ile gerçekleştirilmiştir. DFT(B3LYP, B3PW91) ve HF metodlarıyla 6-311G(d,p) ve 3-21G baz setleri kullanılarak molekülün geometrik, elektronik, spektroskopik ve termodinamik 
parametreleri hesaplanmıştır (Frisch ve ark., 2009; Wolinski ve ark., 1990). Bu aşamada öncelikle, molekülün düşük enerjili, en kararlı üç boyutlu yapısına ulaşmak gereklidir bunun için "Gaussview 5.0" programında (Dennington ve ark., 2009) molekül çizilmiştir ve Gaussian 09W programında seçtiğimiz metod ve temel set ile molekül optimize edilmiştir. Bu en kararlı yapıdan yola çıkarak tüm hesaplamalar yapılmıştır. Karbon ve proton NMR kimyasal kayma değerleri değerleri GIAO metodu ile gaz fazında ve çözücü (DMSO) varlığında hesaplanmış deneysel veriler ile karşılaştırılarak regrasyon analizleri yapılıp grafikleri çizilmiştir. Yine yapı aydınlatılmasında yaygın bir analiz yöntemi olan IR hormonik titreşim frekans değerleri "Veda4f" programı ile hesaplanmıştır (Jamróz, 2004). Elde edilen veriler her metod ve set için belirlenmiş olan skala faktörleri ile çarpılarak skalalı değerlere ulaşılmıştır (Merrick, 2007). Titreşim frekans değerleri kullanılarak teorik IR spektrumları oluşturulmuştur ve deneysel spektrumlar ile karşılaştırılmıştır. Başlıca molekülün geometrik özellikleri (bağ uzunluğu, açısı), elektronegativite $(\chi)$, toplam enerji, atomik yükler, dipol moment $(\mu)$, kimyasal sertlik $(\eta)$ ve yumuşaklık $(S)$, iyonlaşma potansiyeli $(\mathrm{I})$, enerji farkı $(\Delta \mathrm{E} g)$, dönme sabitleri, entropi değerleri, termal enerjisi ve termal kapasitesi gibi özellikleri DFT(B3LYP, B3PW91) ve HF yöntemi ile 6-311G(d,p) ve 3-21G farklı iki temel setde hesaplanmıştır. Ayrıca moleküler elektron potansiyel (MEP), yüzey haritaları (elektron potansiyeli ve elektron yoğunluk potansiyeli), toplam yoğunluk gibi yüzey şekilleri belirlenmiştir.

\section{Bulgular}

\subsection{Geometri Optimizasyonu}

Molekülün geometrik optimizasyonu, gaz ortamında ve temel halde atomların düşük enerjili en kararlı konumlarıdır. Molekülün optimizasyonu DFT(B3LYP, B3PW91) ve HF metodlarıyla 6-311G(d,p) ve 3-21G baz setleri kullanılarak elde edilmiştir ve Şekil.1' de gösterilmiştir. Optimize Gauss-view görüntüsünden geometrik özellikler (bağ açısı ve bağ uzunluğu) bulunmuştur. Bağ uzunlukları ve bağ açılarının literatürde kayıtlı veriler ile kıyaslaması yapılmıştır. DFT (B3LYP, B3PW91), HF için sırası ile 6311G(d,p)/3-21G setlerine göre hesaplanan C1-N24; C1-N26; C2-N25; C2-N26; C5-N27; C6-N27 bağ uzunluklar1 1.331, $1.329,1.296 / 1.347,1.347,1.307 ; 1.365,1.360,1.357 / 1.384,1.381,1.375 ; 1.357,1.353,1.332 / 1.367,1.364,1.345 ; 1.323,1.320$, $1.298 / 1.339,1.338,1.309 ; 1.337,1.334,1.320 / 1.353,1.351,1.332 ; 1.335,1.332,1.317 / 1.349,1.347,1.328 \AA$ olarak bulunmuştur ve Tablo 1'de gösterilmiştir. Literatüre kayıtlı bağ uzunlukları ise $\mathrm{C}=\mathrm{N} ; 1,27 \AA$ ve $\mathrm{C}-\mathrm{N} ; 1.47 \AA$ 'dur. Ayrıca literatürdeki veriler ile kıyaslanacak bir başka bağ uzunlukları ise benzen halkasındaki C-C ve C-H uzunluklarıdır. Moleküldeki benzen halkasının C-C bağ uzunlukları ortalama değerleri B3LYP, B3PW91 ve HF için 6-311G(d,p)/ 3-21G setlerine göre sırası ile 1.39, 1.39, 1.38/ 1.39, 1.39, 1.38 olarak; C-H ortalama bağ uzunluğu ise 1.083, 1.084, 1.074/ 1.082, 1.083, $1.074 \AA$ olarak hesaplanmıştır. Literatürde benzen halkasındaki bağ uzunlukları C-C; $1.39 \AA$ ve C-H; $1.08 \AA$ olarak ölçülmüştür. Bağ uzunlukları verileri kendi aralarında metodlara göre değerlendirildiğinde 6-311G(d,p) ve 3-21G setlerinin her ikisinde DFT fonksiyonları B3LYP, B3PW91 ile hesaplanan bağ uzunluk değerleri kendi aralarında ve literatürle uyumludur.

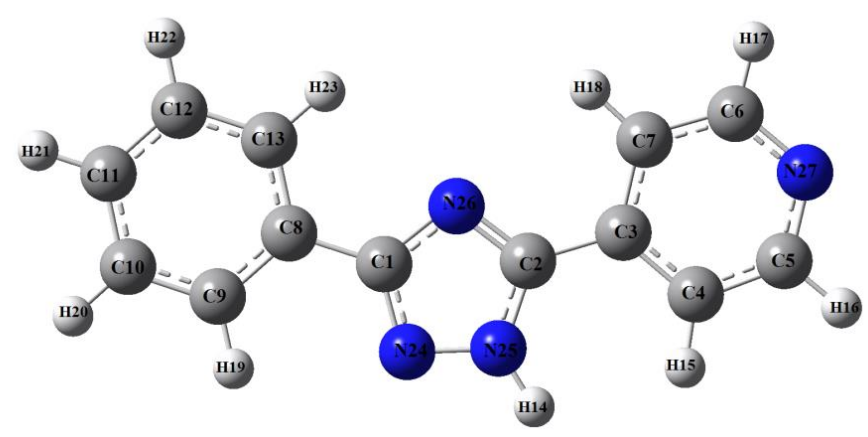

Şekil 1. Bileşiğin B3PW91/6-311G(d,p) seviyesine göre optimize yapr geometrisi 
Tablo 1. Bileşiğin B3LYP, B3PW91 ve HF 6-311G(d,p)/3-21G temel setleri ile hesaplanmış bă̆ uzunluklarl $\left(A^{0}\right)$

\begin{tabular}{|c|c|c|c|c|c|c|}
\hline \multirow[b]{2}{*}{ Bağ Uzunlukları } & \multicolumn{3}{|c|}{ 6-311G(d,p) } & \multicolumn{2}{|c|}{ 3-21G } & \multirow[b]{2}{*}{ HF } \\
\hline & B3LYP & B3PW91 & HF & B3LYP & B3PW91 & \\
\hline $\mathrm{C}(1)-\mathrm{N}(24)$ & 1.331 & 1.329 & 1.296 & 1.347 & 1.347 & 1.307 \\
\hline $\mathrm{N}(24)-\mathrm{N}(25)$ & 1.348 & 1.339 & 1.337 & 1.404 & 1.397 & 1.391 \\
\hline $\mathrm{N}(25)-\mathrm{C}(2)$ & 1.357 & 1.353 & 1.332 & 1.367 & 1.364 & 1.345 \\
\hline $\mathrm{C}(2)-\mathrm{N}(26)$ & 1.323 & 1.320 & 1.298 & 1.339 & 1.338 & 1.309 \\
\hline $\mathrm{C}(3)-\mathrm{C}(4)$ & 1.398 & 1.396 & 1.386 & 1.403 & 1.401 & 1.386 \\
\hline $\mathrm{C}(4)-\mathrm{H}(15)$ & 1.081 & 1.082 & 1.071 & 1.081 & 1.082 & 1.068 \\
\hline $\mathrm{C}(4)-\mathrm{C}(5)$ & 1.389 & 1.387 & 1.382 & 1.390 & 1.389 & 1.379 \\
\hline $\mathrm{C}(5)-\mathrm{H}(16)$ & 1.086 & 1.087 & 1.075 & 1.083 & 1.084 & 1.369 \\
\hline $\mathrm{C}(5)-\mathrm{N}(27)$ & 1.337 & 1.334 & 1.320 & 1.353 & 1.351 & 1.332 \\
\hline $\mathrm{C}(3)-\mathrm{C}(7)$ & 1.399 & 1.396 & 1.386 & 1.401 & 1.400 & 1.385 \\
\hline $\mathrm{C}(8)-\mathrm{C}(9)$ & 1.399 & 1.397 & 1.387 & 1.401 & 1.399 & 1.386 \\
\hline $\mathrm{C}(9)-\mathrm{H}(19)$ & 1.082 & 1.083 & 1.073 & 1.082 & 1.083 & 1.069 \\
\hline $\mathrm{C}(9)-\mathrm{C}(10)$ & 1.391 & 1.389 & 1.384 & 1.393 & 1.392 & 1.382 \\
\hline $\mathrm{C}(10)-\mathrm{H}(20)$ & 1.084 & 1.085 & 1.075 & 1.083 & 1.084 & 1.071 \\
\hline $\mathrm{C}(10)-\mathrm{C}(11)$ & 1.393 & 1.391 & 1.383 & 1.397 & 1.396 & 1.384 \\
\hline $\mathrm{C}(11)-\mathrm{H}(21)$ & 1.084 & 1.085 & 1.075 & 1.083 & 1.084 & 1.071 \\
\hline $\mathrm{C}(11)-\mathrm{C}(12)$ & 1.394 & 1.392 & 1.386 & 1.398 & 1.397 & 1.385 \\
\hline $\mathrm{C}(12)-\mathrm{H}(22)$ & 1.084 & 1.085 & 1.075 & 1.083 & 1.084 & 1.071 \\
\hline $\mathrm{C}(12)-\mathrm{C}(13)$ & 1.389 & 1.387 & 1.381 & 1.392 & 1.391 & 1.381 \\
\hline $\mathrm{C}(13)-\mathrm{H}(23)$ & 1.082 & 1.084 & 1.073 & 1.082 & 1.084 & 1.070 \\
\hline $\mathrm{C}(13)-\mathrm{C}(8)$ & 1.401 & 1.398 & 1.390 & 1.402 & 1.400 & 1.387 \\
\hline
\end{tabular}

Tablo 2. Bileşiğin B3LYP, B3PW91 ve HF 6-311G(d,p)/3-21G temel setleri ile hesaplanmış băg açıları $\left(^{0}\right)$

\begin{tabular}{|c|c|c|c|c|c|c|}
\hline \multirow[b]{2}{*}{ Bağ Açıları } & \multicolumn{3}{|c|}{ 6-311G(d,p) } & \multicolumn{2}{|c|}{ 3-21G } & \multirow[b]{2}{*}{ HF } \\
\hline & B3LYP & B3PW91 & HF & B3LYP & B3PW91 & \\
\hline $\mathrm{N}(24)-\mathrm{C}(1)-\mathrm{N}(26)$ & 113.791 & 113.882 & 113.647 & 114.055 & 114.055 & 113.129 \\
\hline $\mathrm{N}(24)-\mathrm{N}(25)-\mathrm{C}(2)$ & 110.802 & 110.945 & 110.061 & 110.353 & 110.485 & 109.441 \\
\hline $\mathrm{H}(14)-\mathrm{N}(25)-\mathrm{C}(2)$ & 129.846 & 129.694 & 129.978 & 130.809 & 130.775 & 131.220 \\
\hline $\mathrm{N}(25)-\mathrm{C}(2)-\mathrm{C}(3)$ & 125.277 & 125.316 & 125.125 & 126.151 & 126.267 & 125.937 \\
\hline $\mathrm{N}(26)-\mathrm{C}(2)-\mathrm{C}(3)$ & 126.080 & 126.027 & 125.542 & 124.854 & 124.754 & 124.905 \\
\hline $\mathrm{N}(26)-\mathrm{C}(1)-\mathrm{C}(8)$ & 123.415 & 123.438 & 123.006 & 122.861 & 122.912 & 122.734 \\
\hline $\mathrm{C}(1)-\mathrm{C}(8)-\mathrm{C}(9)$ & 120.069 & 120.020 & 120.049 & 119.664 & 119.601 & 119.657 \\
\hline $\mathrm{C}(1)-\mathrm{C}(8)-\mathrm{C}(13)$ & 120.601 & 120.567 & 120.482 & 120.509 & 120.490 & 120.430 \\
\hline $\mathrm{C}(8)-\mathrm{C}(9)-\mathrm{H}(19)$ & 118.961 & 118.909 & 119.345 & 118.601 & 118.604 & 119.022 \\
\hline $\mathrm{H}(19)-\mathrm{C}(9)-\mathrm{C}(10)$ & 120.820 & 120.914 & 120.463 & 121.436 & 121.470 & 121.037 \\
\hline $\mathrm{C}(12)-\mathrm{C}(13)-\mathrm{H}(23)$ & 120.596 & 120.663 & 120.336 & 121.020 & 121.036 & 120.725 \\
\hline $\mathrm{H}(23)-\mathrm{C}(13)-\mathrm{C}(8)$ & 119.191 & 119.166 & 119.447 & 119.016 & 119.049 & 119.299 \\
\hline $\mathrm{C}(2)-\mathrm{C}(3)-\mathrm{C}(4)$ & 119.556 & 119.410 & 119.440 & 118.583 & 118.415 & 118.619 \\
\hline$C(2)-C(3)-C(7)$ & 122.887 & 122.983 & 122.572 & 123.191 & 123.299 & 122.693 \\
\hline $\mathrm{C}(3)-\mathrm{C}(4)-\mathrm{C}(5)$ & 118.805 & 118.741 & 118.430 & 118.872 & 118.822 & 118.656 \\
\hline $\mathrm{C}(3)-\mathrm{C}(4)-\mathrm{H}(15)$ & 119.799 & 119.739 & 120.392 & 119.087 & 119.066 & 119.619 \\
\hline $\mathrm{H}(15)-\mathrm{C}(4)-\mathrm{C}(5)$ & 121.396 & 121.520 & 121.179 & 122.041 & 122.111 & 121.724 \\
\hline $\mathrm{C}(4)-\mathrm{C}(5)-\mathrm{N}(27)$ & 123.980 & 124.046 & 123.790 & 123.401 & 123.437 & 122.627 \\
\hline $\mathrm{C}(4)-\mathrm{C}(5)-\mathrm{H}(16)$ & 120.015 & 119.988 & 119.977 & 120.233 & 120.232 & 120.638 \\
\hline $\mathrm{H}(16)-\mathrm{C}(5)-\mathrm{N}(27)$ & 116.005 & 115.966 & 116.233 & 116.366 & 116.330 & 116.735 \\
\hline $\mathrm{C}(5)-\mathrm{N}(27)-\mathrm{C}(6)$ & 116.896 & 116.846 & 117.582 & 117.272 & 117.243 & 118.771 \\
\hline $\mathrm{N}(27)-\mathrm{C}(6)-\mathrm{H}(17)$ & 116.170 & 116.123 & 116.394 & 116.522 & 116.468 & 116.882 \\
\hline
\end{tabular}

\subsection{Mulliken Atom Yük Verileri}

Molekülün atomlarının mulliken yük değerleri B3LYP ,B3PW91 ve HF metodları ve 6-311G(d,p) ve 3-21G farklı iki temel set ile hesaplanmıştır. Muliken atom yük verileri ile dipol moment değerleri elde edilmiştir. DFT(B3LYP, B3PW91) ve HF 6-311G(d,p)/ 
3-21G ile hesaplama sonuçlarına göre moleküldeki N24, N25, N26, N27 elektronegatif atomlarının mulliken atomik yükleri negatif, moleküldeki tüm protonların mulliken yükleri her iki yönteme ve metoda göre pozitif olarak hesaplanmıştır. Ayrıca "C1, C2, C5, C6" atomları tüm metod ve setlerde pozitif mulliken yük değerindeyken, diğer karbon atomları negatif değerde bulunmuştur. Bunun nedeni, C1, C2 atomlarına komşu iki tane elektronegatif azot atomu , C5, C6 atomlarına komşu bir tane elektronegatif azot atomu vardır.

Tablo 3. Bileşiğin B3LYP, B3PW91 ve HF 6-311G(d,p)/3-21G temel setleri ile hesaplanmış mulliken atom yükleri

\begin{tabular}{|c|c|c|c|c|c|c|}
\hline \multicolumn{4}{|c|}{ 6-311G(d,p) } & \multicolumn{3}{|c|}{ 3-21G } \\
\hline & B3LYP & B3PW91 & HF & B3LYP & B3PW91 & HF \\
\hline C1 & 0.290 & 0.339 & 0.477 & 0.485 & 0.491 & 0.637 \\
\hline $\mathrm{C2}$ & 0.438 & 0.486 & 0.595 & 0.656 & 0.668 & 0.835 \\
\hline $\mathbf{C 3}$ & -0.102 & -0.117 & -0.082 & -0.016 & -0.039 & -0.082 \\
\hline C4 & -0.108 & -0.126 & -0.182 & -0.217 & -0.240 & -0.285 \\
\hline C5 & 0.076 & 0.094 & 0.169 & 0.072 & 0.051 & 0.131 \\
\hline C6 & 0.070 & 0.087 & 0.166 & 0.078 & 0.057 & 0.137 \\
\hline C7 & -0.127 & -0.146 & -0.206 & -0.246 & -0.269 & -0.313 \\
\hline C8 & -0.196 & -0.234 & -0.225 & -0.055 & -0.279 & -0.161 \\
\hline C9 & -0.021 & -0.019 & -0.060 & -0.182 & -0.203 & -0.207 \\
\hline C10 & -0.097 & -0.108 & -0.081 & -0.182 & -0.213 & -0.235 \\
\hline C11 & -0.080 & -0.088 & -0.093 & -0.182 & -0.212 & -0.234 \\
\hline $\mathrm{C} 12$ & -0.095 & -0.106 & -0.079 & -0.182 & -0.213 & -0.235 \\
\hline $\mathrm{C13}$ & -0.026 & -0.026 & -0.058 & -0.187 & -0.207 & -0.209 \\
\hline H14 & 0.243 & 0.252 & 0.253 & 0.353 & 0.373 & 0.402 \\
\hline H15 & 0.119 & 0.131 & 0.128 & 0.227 & 0.258 & 0.296 \\
\hline H16 & 0.116 & 0.127 & 0.117 & 0.218 & 0.247 & 0.266 \\
\hline H17 & 0.113 & 0.123 & 0.114 & 0.216 & 0.244 & 0.264 \\
\hline H18 & 0.079 & 0.090 & 0.083 & 0.175 & 0.205 & 0.237 \\
\hline H19 & 0.103 & 0.115 & 0.111 & 0.209 & 0.240 & 0.275 \\
\hline H20 & 0.095 & 0.105 & 0.098 & 0.188 & 0.219 & 0.242 \\
\hline H21 & 0.095 & 0.105 & 0.098 & 0.188 & 0.218 & 0.242 \\
\hline H22 & 0.095 & 0.105 & 0.098 & 0.188 & 0.219 & 0.242 \\
\hline $\mathrm{H} 23$ & 0.099 & 0.110 & 0.107 & 0.206 & 0.237 & 0.271 \\
\hline N24 & -0.212 & -0.235 & -0.291 & -0.320 & -0.321 & -0.380 \\
\hline N25 & -0.298 & -0.322 & -0.358 & -0.587 & -0.611 & -0.746 \\
\hline N26 & -0.383 & -0.424 & -0.513 & -0.583 & -0.590 & -0.731 \\
\hline N27 & -0.285 & -0.318 & -0.386 & -0.521 & -0.531 & -0.659 \\
\hline
\end{tabular}

\subsection{HOMO-LUMO Enerjileri ve Elektronik Özellikleri}

Frontier Moleküler orbitalleri (FMO) olan Homo-Lumo kinetik kararlılığı, elektron geçişlerini, elektrik ve optik özellikleri açıklar. HOMO yüksek enerjili dolu moleküler orbitali, LUMO ise düşük enerjili boş moleküler orbitali ifade eder. Ayrıca HOMO elektron veren, LUMO ise elektron alan ifadelerini karşılar ve bu iki orbitalin enerji boşluğu " $\Delta \mathrm{Eg}$ " kinetik kararlılık için çok önemlidir. " $\Delta \mathrm{E} g$ " enerji farkı ne kadar düşükse molekül daha kararlıdır. Molekülün B3LYP, B3PW91, HF 6-311G(d,p)/ 3-21G ile HOMO-LUMO enerjileri teorik olarak bulunmuştur. B3LYP/6-311G(d,p) için $\Delta \mathrm{E} g=4.4695 \mathrm{eV}, \mathrm{B} 3 \mathrm{PW} 91 / 6-311 \mathrm{G}(\mathrm{d}, \mathrm{p})$ için $\Delta \mathrm{E} g=4.5025 \mathrm{eV}, \mathrm{HF} / 6-311 \mathrm{G}(\mathrm{d}, \mathrm{p})$ için $\Delta \mathrm{E} g=10.4048 \mathrm{eV}$; B3LYP/ 3-21G için $\Delta \mathrm{E} g=4.5936 \mathrm{eV}$, B3PW91/ 3-21G için $\Delta \mathrm{E} g=4.6135$ $\mathrm{eV}, \mathrm{HF} / 3-21 \mathrm{G}$ için $\Delta \mathrm{Eg}=10.6339 \mathrm{eV}$ olarak hesaplanmıştır ve Şekil 2, 3'de gösterilmiştir. Ayrıca elde edilen HOMO-LUMO enerji farkından faydalanarak molekülün tüm elektronik parametreleri hesaplanmıştır (Tablo 4).

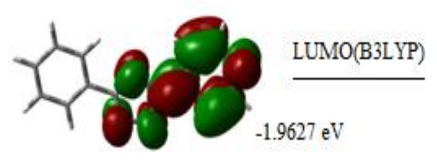

$\Delta \mathrm{E} g=4.4695 \mathrm{eV}$

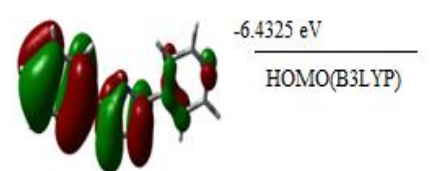

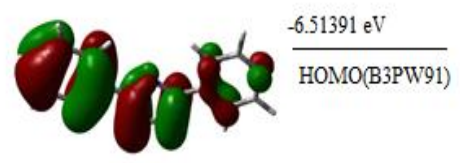

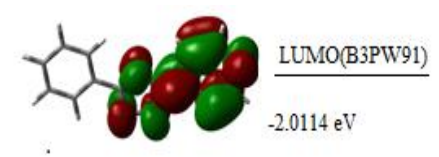

$\Delta \mathrm{Eg}=4.5025 \mathrm{eV}$

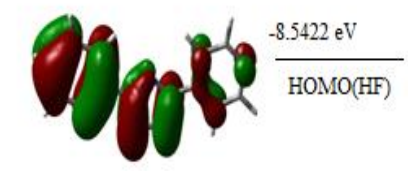

Şekil 2. Bileşiğin B3LYP, B3PW91, HF 6-311G(d,p) seviyesine göre HOMO-LUMO enerjileri 


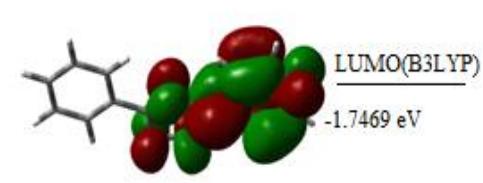

$\Delta \mathrm{E} g=4.5936 \mathrm{eV}$

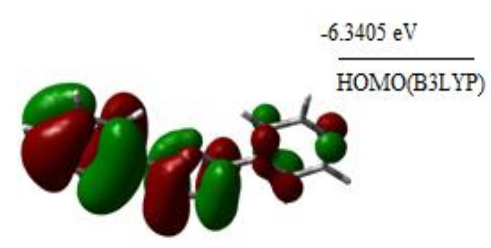

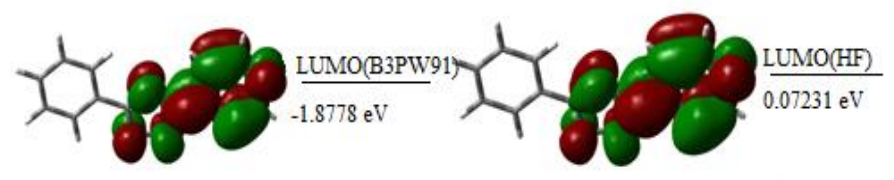

$\Delta \mathrm{E} g=4.6135 \mathrm{eV}$

$\Delta \mathrm{E} g=10.6339 \mathrm{eV}$
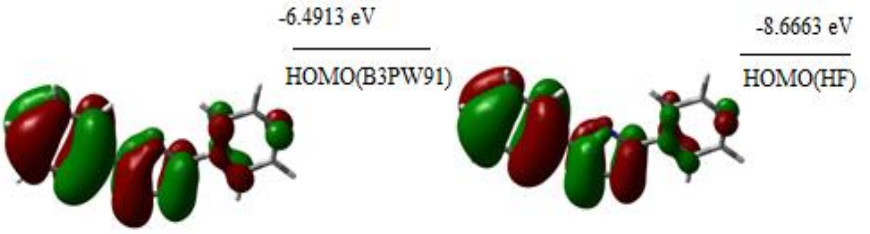

Şekil 3. Bileşiğin B3LYP, B3PW91, HF 3-21G seviyesine göre HOMO-LUMO enerjileri

Tablo 4. Bileşiğin B3LYP, B3PW91, HF 6-311G(d,p)/3-21G temel setleri ile hesaplanmış elektronik özellikleri

\begin{tabular}{|c|c|c|c|c|c|c|c|}
\hline & & $\begin{array}{c}\text { B3LYP } \\
\text { 6-311G(d,p) }\end{array}$ & $\begin{array}{c}\text { B3PW91 } \\
\text { 6-311G(d,p) }\end{array}$ & $\begin{array}{c}\text { HF } \\
6-311 G(d, p)\end{array}$ & $\begin{array}{l}\text { B3LYP } \\
\text { 3-21G }\end{array}$ & $\begin{array}{c}\text { B3PW91 } \\
\text { 3-21G }\end{array}$ & $\begin{array}{c}\text { HF } \\
\text { 3-21G }\end{array}$ \\
\hline & & $\mathrm{eV}$ & $\mathrm{eV}$ & $\mathrm{eV}$ & eV & eV & eV \\
\hline & LUMO & $-1,96271$ & $-2,01141$ & 1,86257 & $-1,74693$ & $-1,87781$ & 1,96761 \\
\hline & HOMO & $-6,43234$ & $-6,5137$ & $-8,54198$ & $-6,34037$ & $-6,49111$ & $-8,66606$ \\
\hline $\mathbf{A}$ & Elektron ilgisi & 1,96271 & 2,01141 & $-1,86257$ & 1,74693 & 1,87781 & $-1,96761$ \\
\hline I & İyonlaşma potansiyeli & 6,43234 & 6,5137 & 8,54198 & 6,34037 & 6,49111 & 8,66606 \\
\hline$\Delta \mathbf{E}$ & Enerji fark1 & 4,46963 & 4,50228 & 10,4046 & 4,59344 & 4,6133 & 10,6337 \\
\hline$\chi$ & Elektronegativite & 4,19752 & 4,26256 & 3,33971 & 4,04365 & 4,18446 & 3,34923 \\
\hline$\stackrel{\tilde{P}}{\mathbf{P}}$ & Kimyasal potansiyel & $-4,19752$ & $-4,26256$ & $-3,33971$ & $-4,04365$ & $-4,18446$ & $-3,34923$ \\
\hline$\omega$ & Elektrofilik indeks & 0,02659 & 0,02762 & 0,03918 & 0,02536 & 0,02727 & 0,04027 \\
\hline IP & Nükleofilik indeks & $-0,34474$ & $-0,35264$ & $-0,6385$ & $-0,3413$ & $-0,35472$ & $-0,65442$ \\
\hline $\mathbf{S}$ & Moleküler yumuşaklık & 331,313 & 328,91 & 142,327 & 322,383 & 320,994 & 139,26 \\
\hline$\eta$ & Moleküler sertlik & 2,23481 & 2,25114 & 5,20228 & 2,29672 & 2,30665 & 5,31683 \\
\hline
\end{tabular}

\subsection{Termodinamik Özellikler}

Başlıca molekülün B3LYP, B3PW91, HF 6-311G(d,p)/ 3-21G ile termodinamik parametreleri hesaplanmıştır. Bir kimyasal tepkimenin gerçekleşip gerçekleşmediği hakkında bilgi edinmemize termodinamik 1sı etkileri katk1 sağlar. Bu sebeple organik bileşiklerin reaksiyon mekanizmalarını incelemek için bu termodinamik verilerden faydalanılır. Reaksiyonların 1sı oluşum değerlerini belirlemek için kuantum kimyasal hesaplamalar kullanılmıştır ve Dönme sabitleri (GHz), Entropi: S (cal/molK), Termal Enerji: $\mathrm{E}(\mathrm{Kcal} / \mathrm{mol})$, Termal Kapasite: $\mathrm{CV}(\mathrm{Cal} / \mathrm{Mol}-\mathrm{Kel}$ vin) değerleri bulunmuştur (Tablo 5).

Tablo 5. Bileşiğin B3LYP, B3PW91/6-311G(d,p)/3-21G temel setleri ile hesaplanmış termodinamik özellikleri

\begin{tabular}{|c|c|c|c|c|c|c|}
\hline Parametreler & $\begin{array}{l}\text { B3LYP/ } \\
\text { 6-311G(d,p) }\end{array}$ & $\begin{array}{l}\text { B3PW91/ } \\
\text { 6-311G(d,p) }\end{array}$ & $\begin{array}{l}\text { HF/ } \\
6-311 G(d, p)\end{array}$ & $\begin{array}{l}\text { B3LYP/ } \\
\text { 3-21G }\end{array}$ & $\begin{array}{l}\text { B3PW91/ } \\
\text { 3-21G }\end{array}$ & $\begin{array}{l}\text { HF/ } \\
\text { 3-21G }\end{array}$ \\
\hline Sıfır nokta titreşim enerjisi (Kcal/mol) & 131.2880 & 131.8256 & 140.8077 & 132.2233 & 132.6497 & 142.6072 \\
\hline Toplam enerji (a.u) & 720.5840 & 720.2975 & 716.0624 & 716.4189 & 716.1450 & 711.8797 \\
\hline \multicolumn{7}{|l|}{ Dönme sabitleri (GHz) } \\
\hline A & 1.69438 & 1.70207 & 1.72017 & 1.63275 & 1.63627 & 1.66610 \\
\hline B & 0.20371 & 0.20471 & 0.20629 & 0.20490 & 0.20567 & 0.20811 \\
\hline C & 0.18184 & 0.18273 & 0.18420 & 0.18206 & 0.18270 & 0.18500 \\
\hline \multicolumn{7}{|l|}{ Entropi: S (cal/molK) } \\
\hline Dönme & 42.096 & 42.096 & 42.096 & 42.096 & 42.096 & 42.096 \\
\hline Ötelenme & 32.904 & 32.890 & 32.864 & 32.934 & 32.925 & 32.883 \\
\hline Titreşim & 41.017 & 41.050 & 31.518 & 38.364 & 38.586 & 35.620 \\
\hline Toplam & 116.018 & 116.036 & 106.478 & 113.394 & 113.607 & 110.599 \\
\hline \multicolumn{7}{|l|}{ Termal Enerji: E(Kcal/mol) } \\
\hline Dönme & 0.889 & 0.889 & 0.889 & 0.889 & 0.889 & 0.889 \\
\hline Ötelenme & 0.889 & 0.889 & 0.889 & 0.889 & 0.889 & 0.889 \\
\hline Titreşim & 137.436 & 137.970 & 145.883 & 138.179 & 138.608 & 147.991 \\
\hline Toplam & 139.213 & 139.748 & 147.661 & 139.956 & 140.386 & 149.768 \\
\hline \multicolumn{7}{|l|}{ Termal Kapasite: CV(Cal/Mol-Kelvin) } \\
\hline Dönme & 2.981 & 2.981 & 2.981 & 2.981 & 2.981 & 2.981 \\
\hline Ötelenme & 2.981 & 2.981 & 2.981 & 2.981 & 2.981 & 2.981 \\
\hline Titreşim & 43.651 & 43.528 & 37.735 & 42.767 & 42.643 & 38.180 \\
\hline Toplam & 49.613 & 49.489 & 43.696 & 48.729 & 48.605 & 44.142 \\
\hline
\end{tabular}




\subsection{Yüzey Haritaları}

B3LYP, B3PW91, HF 6-311G(d,p)/ 3-21G metodlar ve temel setler kullanılarak molekülün üç boyutlu yüzey haritaları belirlenmiş̧tir. MEP ' in üç boyutlu şekilleri Moleküler elektrostatik potansiyel haritası olarak bilinmektedir. MEP haritasının yüzeyinde moleküler yüzey, boyut, yük dağılımı, elektrostatik potansiyel değerleri gösterilmiştir. Molekülde var olan elektronegatif atomların çevresi kırmızı, elektropozitif atomların çevresi mavi ve diğer atomların çevreleri ise yeşil renktedir. Molekülde elektron yoğunluğunun en yüksek olduğu bölge N26, N27 atomlarının çevresidir, elektron yoğunluğunun en düşük olduğu bölge ise N-H protonunun çevresidir. Ayrıca, toplam yoğunluk ve yüzey haritaları belirlenerek Şekil 4'de gösterilmiş̧ir.

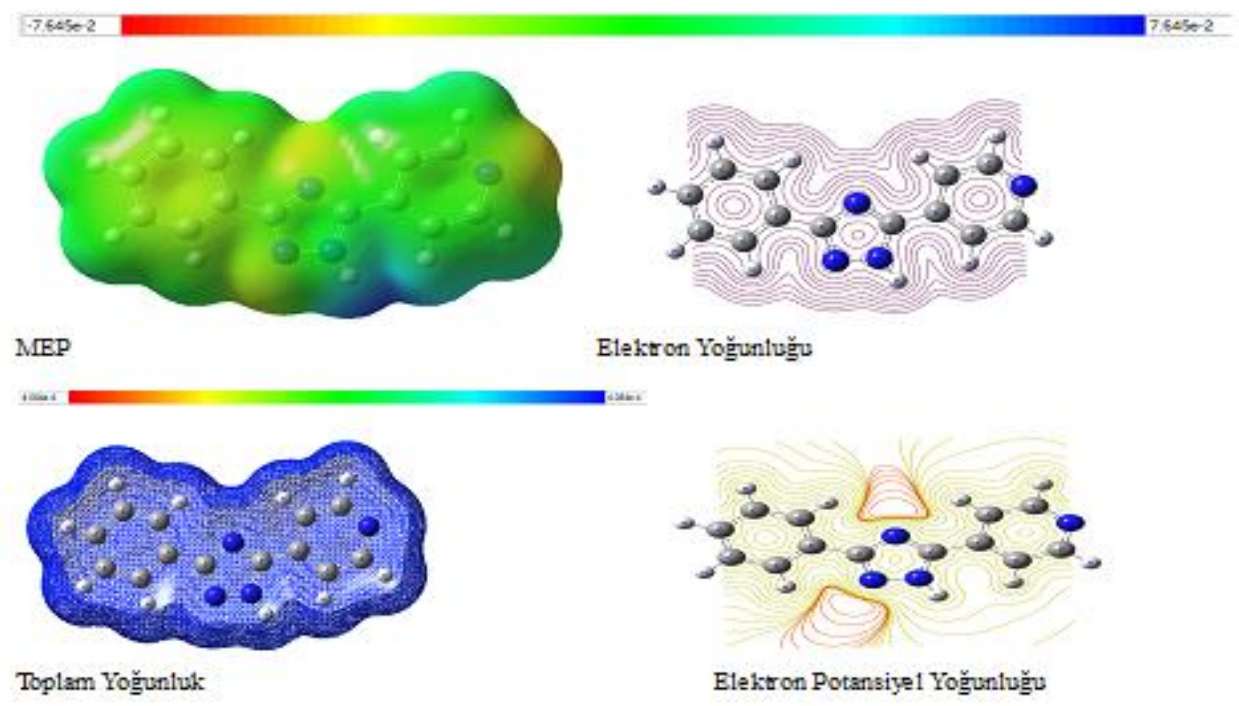

Şekil 4. Bileşiğin B3PW91/ 6-311G(d,p) seviyesine göre yüzey haritalarl

\subsection{IR Analizi}

Molekülün Infrared titreşim frekans değerleri B3LYP, B3PW91, HF 6-311G(d,p) temel setleri ile hesaplanmıştır ve elde edilen veriler uygun skala faktörleri ile çarpılarak skalalı değerlere ulaşılmıştır. Deneysel N-H gerilme titreşimi $3180 \mathrm{~cm}^{-1}$ 'de, B3LYP /6$311 \mathrm{G}(\mathrm{d}, \mathrm{p})$ ile $3541 \mathrm{~cm}^{-1} \mathrm{de}$, B3PW91/6-311G(d,p) ile $3557 \mathrm{~cm}^{-1} \mathrm{de}, \mathrm{HF} / 6-311 \mathrm{G}(\mathrm{d}, \mathrm{p})$ ile $3553 \mathrm{~cm}^{-1}$ de; deneysel $\mathrm{C}=\mathrm{N}$ gerilme titreşimi

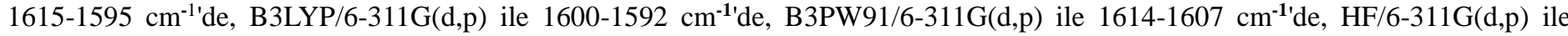
1644-1634 $\mathrm{cm}^{-1}$ 'de; benzen halkasındaki aromatik deneysel C-H gerilme titreşimleri $755-695 \mathrm{~cm}^{-1}$ de, B3LYP/6-311G(d,p) ile 735$688 \mathrm{~cm}^{-1}$ 'de, B3PW91/6-311G(d,p) ile 736-689 $\mathrm{cm}^{-1}$ 'de, HF/6-311G(d,p) ile 755-695 cm-1'de gözlenmiştir ve Tablo 6' da gösterilmiştir. Ayrıca teorik olarak infrared spektrumları çizilmiştir ve Şekil 5'de gösterilmiştir. 
Tablo 6. Molekülün seçilmiş titreşim frekans değerleri ve türleri

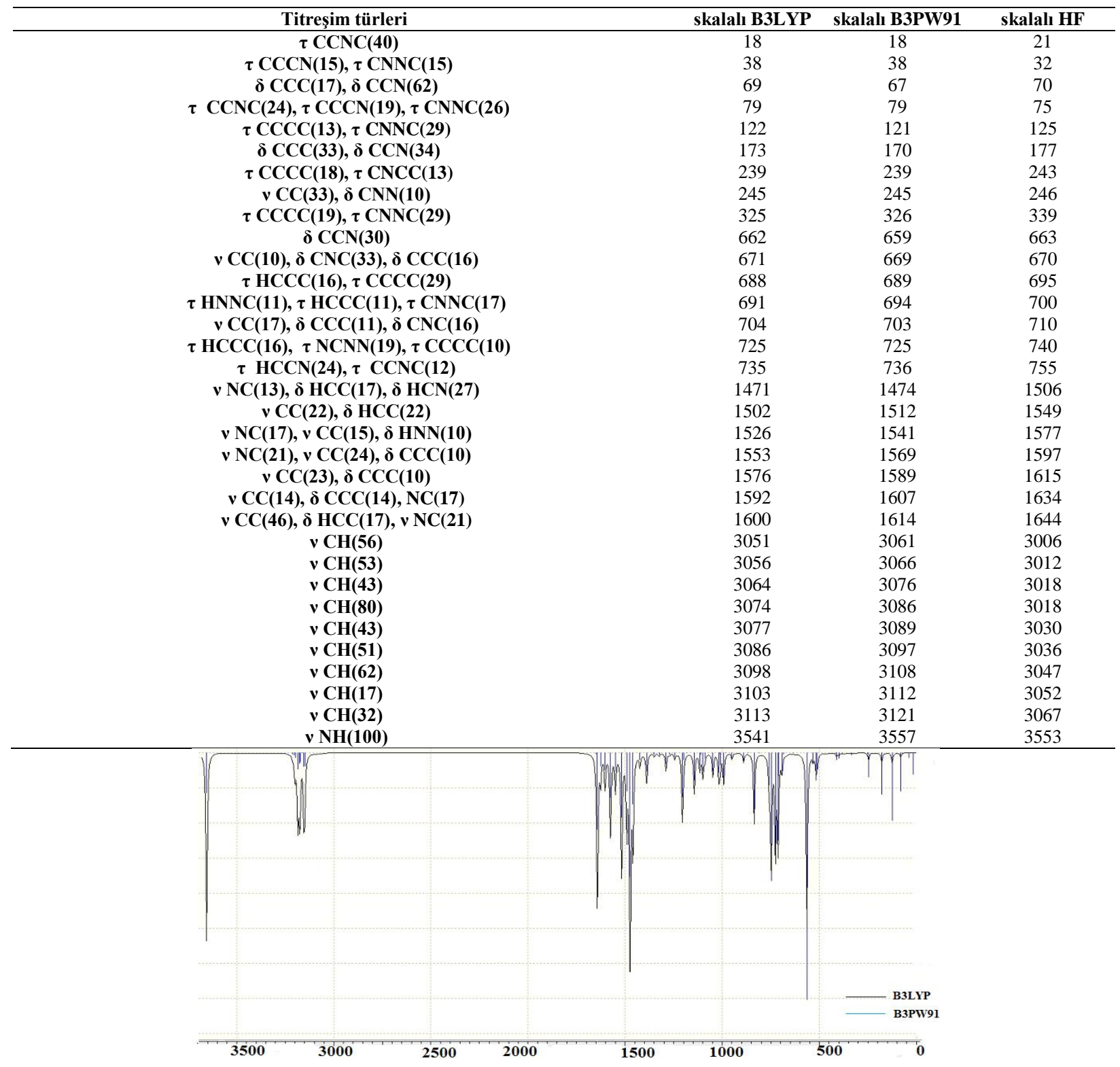

Şekil 5. Bileşiğin B3PW91/ B3LYP 6-311G(d,p) seviyesine göre IR Spektrumlart

\subsection{NMR Spektral Analizi}

3-Fenil-5-(4-pridil)-1,2,4-triazol bileşiğinin B3LYP, B3PW91, HF 6-311G(d,p)/ 3-21G ile ${ }^{13} \mathrm{C} /{ }^{1} \mathrm{H}-\mathrm{NMR}$ teorik kimyasal kayma değerleri gaz ortamında ve çözücülü (DMSO) ortamda hesaplanmıştır ve literatürden alınan deneysel değerler ile karşılaştırılarak Tablo 7'de gösterilmiştir. Moleküldeki benzen halkasının protonlarının (H19-H23) deneysel kimyasal kayma değerleri 7.50-8.20 ppm aralığında, piridin halkasının protonları (H15-H18) 8.26-8.48 ppm aralığındadır ve N-H protonunun (H14) kimyasal kayma değeri 14.30 ppm' dir. B3LYP, B3PW91, HF 6-311G(d,p) temel seti ile elde edilen teorik ${ }^{1} \mathrm{H}$ kimyasal kayma değerleri benzen halkasının protonları (H19-H23) için gaz ortamında sırası ile 8.11-9.30, 8.29-9.49, 7.13-8.35 ppm aralığında, çözücülü (DMSO) ortamda 7.598.58, 8.49-9.49, 8.07- 9.08 ppm aralığındadır, piridin halkasının protonları (H15-H18) gaz ortamında sırası ile 7.89-9.72, 8.06-9.87, 6.88-8.62 ppm aralığında iken, çözücülü (DMSO) ortamda 7.71-8.95, 8.62-9.87, 8.16-9.36 ppm aralığındadır, N-H protonunun (H14) teorik kimyasal kayma değeri gaz ortamında 10.65, 10.73, 9.78 ppm iken, çözücülü (DMSO) ortamda 10.54, 11.35, 10.47 ppm'dir. ${ }^{13} \mathrm{C}-\mathrm{NMR}$ deneysel analizinde benzen halkasının karbonları (C8-C13) 127.98-135.00 ppm'de, Piridin halkasının karbonları (C3- C7) 121.60-154-70 ppm' de, Triazol halkasının karbonları (C1, C2) 160.71, 158.20 ppm'de gözlemlenmiştir. B3LYP, B3PW91, HF 6$311 \mathrm{G}(\mathrm{d}, \mathrm{p})$ temel seti ile gaz ortamında hesaplanan benzen halkasının karbonları (C8-C13) için ${ }^{13} \mathrm{C}-\mathrm{NMR}$ verileri ise sırası ile; 129.75-135.72, 125.85-130.30, 119.87-122.40 ppm'de, çözücülü ortamda ise 128.97-135.19, 125.09-130.25, 119.39-123.60 ppm'dir. B3LYP, B3PW91, HF 6-311G(d,p) temel seti ile gaz ortamında hesaplanan piridin halkasının karbonları (C3-C7) 118.15-156.15, 114.13-151.97, 106.55-144.85 ppm'dir, çözücülü (DMSO)ortamda ise 121.43-155.15, 117.39-151.35, 109.52-143.95 ppm'dir. B3LYP, B3PW91, HF 6-311G(d,p) temel seti ile gaz ortamında hesaplanan Triazol halkasının karbonlar1 (C1, C2) 168.23, 156.15; 162.77, 150,83; 153.76, 145.77 ppm'dir, çözücülü (DMSO) ortamda ise 167.28, 157.37; 161.77, 152.00; 153.12, 147.13 ppm'dir. Deneysel 
değerler ile teorik değerleri mukayese etmek için regrasyon analizleri yapılarak $\mathrm{R}^{2}$ (ortalama küresel hata) değerleri bulunarak grafikler oluşturulmuştur ve Şekil 6' da gösterilmiştir.

Tablo 7. Bileşiğin deneysel ve B3LYP, B3PW91/6-311G(d,p) temel setleri ile hesaplanmış ${ }^{1} \mathrm{H} /{ }^{13} \mathrm{C}-\mathrm{NMR}$ verileri

\begin{tabular}{|c|c|c|c|c|c|c|c|c|c|c|c|c|c|}
\hline No & Deney. & $\begin{array}{l}\text { B3LYP } \\
\text { Vakum }\end{array}$ & $\begin{array}{c}\text { B3LYP } \\
\text { Fark } \\
\text { Vakum }\end{array}$ & $\begin{array}{l}\text { B3LYP } \\
\text { DMSO }\end{array}$ & $\begin{array}{c}\text { Fark } \\
\text { B3LYP } \\
\text { DMSO }\end{array}$ & $\begin{array}{c}\text { B3PW91 } \\
\text { vakum }\end{array}$ & $\begin{array}{c}\text { Fark } \\
\text { B3PW91 } \\
\text { Vakum }\end{array}$ & $\begin{array}{c}\text { B3PW91 } \\
\text { DMSO }\end{array}$ & $\begin{array}{c}\text { Fark } \\
\text { B3PW91 } \\
\text { DMSO }\end{array}$ & $\begin{array}{c}\text { HF } \\
\text { Vakum }\end{array}$ & $\begin{array}{c}\text { Fark } \\
\text { HF } \\
\text { Vakum }\end{array}$ & $\begin{array}{c}\text { HF } \\
\text { DMSO }\end{array}$ & $\begin{array}{c}\text { Fark } \\
\text { HF } \\
\text { DMSO }\end{array}$ \\
\hline C1 & 160.71 & 168.23 & -7.52 & 167.28 & -6.57 & 162.77 & -2.06 & 161.77 & -1.06 & 153.76 & 6.95 & 153.12 & 7.59 \\
\hline $\mathrm{C2}$ & 158.20 & 156.15 & 2.05 & 157.37 & 0.83 & 150.83 & 7.37 & 152.00 & 6.2 & 145.77 & 12.43 & 147.13 & 11.07 \\
\hline C3 & 140.90 & 136.98 & 3.92 & 137.47 & 3.43 & 132.06 & 8.84 & 132.53 & 8.37 & 129.02 & 11.88 & 129.39 & 11.51 \\
\hline C4 & 127.52 & 123.71 & 3.81 & 123.87 & 3.65 & 119.78 & 7.74 & 119.93 & 7.59 & 112.07 & 15.45 & 111.93 & 15.59 \\
\hline C5 & 154.70 & 156.15 & -1.45 & 155.5 & -0.8 & 151.97 & 2.73 & 151.35 & 3.35 & 144.85 & 9.85 & 144.36 & 10.34 \\
\hline C6 & 151.92 & 154.53 & -2.61 & 155.15 & -3.23 & 150.33 & 1.59 & 151.00 & 0.92 & 143.14 & 8.78 & 143.95 & 7.97 \\
\hline C7 & 121.60 & 118.15 & 3.45 & 121.43 & 0.17 & 114.13 & 7.47 & 117.39 & 4.21 & 106.55 & 15.05 & 109.52 & 12.08 \\
\hline C8 & 135.00 & 135.22 & -0.22 & 135.19 & -0.19 & 130.30 & 4.7 & 130.24 & 4.76 & 122.40 & 12.60 & 122.27 & 12.73 \\
\hline C9 & 129.40 & 130.76 & -1.36 & 129.88 & -0.48 & 126.80 & 2.6 & 125.94 & 3.46 & 121.44 & 7.96 & 120.56 & 8.84 \\
\hline C10 & 130.68 & 131.69 & -1.01 & 132.53 & -1.85 & 127.72 & 2.96 & 128.61 & 2.07 & 119.97 & 10.71 & 120.96 & 9.72 \\
\hline C11 & 133.60 & 132.96 & 0.64 & 133.65 & -0.05 & 128.99 & 4.61 & 129.72 & 3.88 & 122.94 & 10.66 & 123.6 & 10 \\
\hline $\mathrm{C} 12$ & 129.77 & 131.57 & -1.8 & 132.45 & -2.68 & 127.60 & 2.17 & 128.53 & 1.24 & 119.87 & 9.90 & 120.87 & 8.9 \\
\hline C13 & 127.98 & 129.75 & -1.77 & 128.97 & -0.99 & 125.85 & 2.13 & 125.09 & 2.89 & 120.33 & 7.65 & 119.39 & 8.59 \\
\hline H14 & 14.30 & 10.65 & 3.65 & 10.54 & 3.76 & 10.73 & 3.57 & 11.35 & 2.95 & 9.08 & 5.22 & 10.47 & 3.83 \\
\hline H15 & 8.26 & 9.09 & -0.83 & 8.4 & -0.14 & 9.29 & -1.03 & 9.31 & -1.05 & 8.17 & 0.09 & 8.89 & -0.63 \\
\hline H16 & 8.48 & 9.72 & -1.24 & 9 & -0.52 & 9.87 & -1.39 & 9.87 & -1.39 & 8.62 & -0.14 & 9.36 & -0.88 \\
\hline H17 & 8.48 & 9.59 & -1.11 & 8.95 & -0.47 & 9.74 & -1.26 & 9.82 & -1.34 & 8.49 & -0.01 & 9.32 & -0.84 \\
\hline H18 & 8.36 & 7.89 & 0.47 & 7.71 & 0.65 & 8.06 & 0.3 & 8.62 & -0.26 & 6.88 & 1.48 & 8.16 & 0.2 \\
\hline H19 & 8.20 & 9.30 & -1.1 & 8.58 & -0.38 & 9.49 & -1.29 & 9.49 & -1.29 & 8.35 & -0.15 & 9.08 & -0.88 \\
\hline H20 & 7.85 & 8.22 & -0.37 & 7.69 & 0.16 & 8.40 & -0.55 & 8.59 & -0.74 & 7.17 & 0.68 & 8.11 & -0.26 \\
\hline H21 & 7.50 & 8.11 & -0.61 & 7.59 & -0.09 & 8.29 & -0.79 & 8.49 & -0.99 & 7.14 & 0.36 & 8.08 & -0.58 \\
\hline H22 & 7.85 & 8.16 & -0.31 & 7.63 & 0.22 & 8.34 & -0.49 & 8.54 & -0.69 & 7.13 & 0.72 & 8.07 & -0.22 \\
\hline H23 & 8.20 & 9.20 & -1 & 8.5 & -0.3 & 9.39 & -1.19 & 9.41 & -1.21 & 8.26 & -0.06 & 8.98 & -0.78 \\
\hline
\end{tabular}
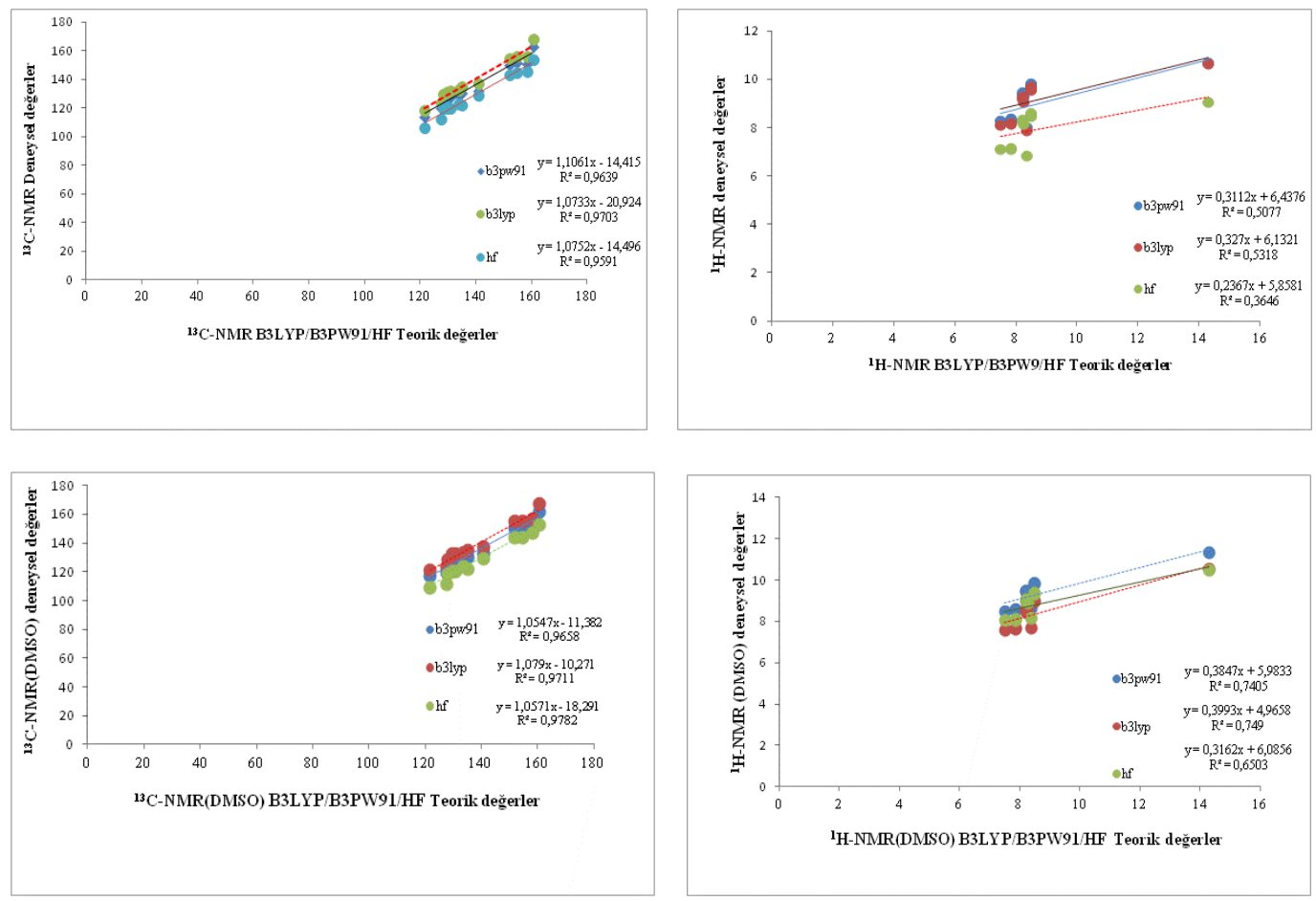

Şekil 6. Bileşiğin gaz ortamında ve çözücülü (DMSO) ortamda regrasyon analiz grafikleri ve $R^{2}$ değerleri

\section{Sonuç}

Çalışmada, 3-Fenil-5-(4-pridil)-1,2,4-triazol bileşiğinin B3LYP, B3PW91, HF 6-311G(d,p)/ 3-21G setleri ile spektroskopik, elektronik, termodinamik ve geometrik özellikleri teorik olarak incelenmiştir. Optimize yapıdan yola çıkarak kapsamlı ve karşılaştırmalı bir şekilde yapılan hesaplamalar sonucunda, molekülün ${ }^{13} \mathrm{C}-\mathrm{NMR}$ verileri için, HF 6-311G (d,p) temel seti ile DMSO çözücülü ortamda $\mathrm{R}^{2}$ değerleri 0.9782 ' dir ve diğer setlerle kıyaslandığında 1'e en yakın değer budur. Bu da gösteriyorki, HF/6-311G (d,p) temel seti ile ${ }^{13} \mathrm{C}-\mathrm{NMR}$ (DMSO) sonuçları deneysele daha yakındır. ${ }^{1} \mathrm{H}-\mathrm{NMR}$ kimyasal kayma sonuçlarında ise grafiklerden anlaşıldığı üzere bir sapma gözlenmiştir. Bunun nedeni yapıda bulunan N-H asidik protonudur. IR titreşim frekansları yine iki farklı metot ve yöntemle teorik olarak hesaplanmıştır ve değerler uygun katsayılar ile çarpılarak deneysel değerlerle karşılaştırılmıştır ve kapsamlı bir set olan B3LYP/6-311G(d,p) ile hesaplanan değerlerin deneysel ile daha uyumlu olduğu sonucuna varılmıştır. Teorik IR verilerinde negatif değerlere rastlanmamıştır bu da bize molekülün kararlı olduğunu göstermiştir. Molekülün HOMO-LUMO 
orbitallerinin enerji değerleri arasında en düşük $\Delta \mathrm{Eg}$ değeri B3LYP/ 6-311G((d,p) ile elde edilen 4.4695 eV'dur ve bu sonuç da yapının kararlı olduğunu bize söyler. Ayrıca, HOMO-LUMO orbital enerjilerini kullanarak elektron ilgisi, iyonlaşma potansiyeli, nükleofilik özellikler, moleküler sertlik-yumuşaklık hesaplanmıştır. Molekülün termodinamik değerleri bulunmuştur, geometrik parametreleri hesaplanmıştır ve bağ uzunlukları literatürdeki değerler ile karşılaştırılmıştır. Bu karşılaştırma sonucunda 6-311G(d,p) setinin DFT (B3LYP ve B3PW91) fonksiyonlari ile elde edilen verilerin kendi aralarinda ve deneysel veriler ile uyumu görülmüştür. Ayrıca, moleküler yüzey haritaları oluşturulmuş ve MEP haritasından molekülün nükleofilik ve elektrofilik bölgeleri tespit edilmiştir.

\section{Kaynakça}

Bahçeci, Ş., Yüksek, H., Serdar, M. (2005). Reactions of amidines with some carboxylic acid hydrazides. Indian Journal of Chemistry, Volum 44B, pp 568-572.

Barton, D., Ollis., W.D. (1979). Comprehensive Organic Chemistry, vol. 2. (Oxford:Pergamon).

Becke, A.D. (1988). Density-functional exchange-energy approximation with correctasymptotic behavior. Physical review A: General physics, 38(6), 3098-3100.

Becke, A.D. (1993). Density-functional thermochemistry III. The role of exact Exchange. The Journal of Chemical Physics, 98, 372377.

Brown, E.J. \& Polya, J.B. (1962). J.Chem. Soc, 5149.

Buzdar, A.U. (2002). Anastrozole (Arimidex ${ }^{\mathrm{TM}}$ ) in clinical practice versus the old 'gold standard'. tamoxifen Expert Rev. anticancer Ther., 2 pp. 623-629.

Casaszar, J., Morvay, J., Herczeg, O. (1985). Acta Phys.Chem., 31, 717-722.

Cohn, M.A., Morris., D.D., Juan, D. (1992). Effects of estazolam and flurazepam on cardiopulmonary function in patients with chronic obstructive pulmonary disease Drug Saf, 7 pp, 152-158

Cozzi, P.G. (2004). Chem. Soc. Rev. 33 (7), 410-421.

Dennington, R., Keith, T., Millam, J. (2009). GaussView. Version 5. Semichem Inc. Shawnee Mission KS.

Fletcher, R.A., Gilley, A., Sankhla, N., Davis, T.D. (2010). Triazoles as plant growth regulators and stress protectants, Hortic. Rev., 24 pp, 55-138.

Frisch, M.J., Trucks, G.W., Schlegel, HB., Scuseria, GE., Robb, MA., Mennucci, B., Petersson, GA., Nakatsuji, H., Caricato, M., Li, X. et al. (2009). Gaussian 09. Revision C.01. Gaussian. Inc. Wallingford. CT.

Graci, J.D., Cameron, C.E. (2002). Quasispecies. error catastrophe and the antiviral activity of ribavirin Virology, 298, pp. 175-180.

Gupta, K., Sutar, A.K., (2008). Coord.Chem. Rev., 252 (12), 1420-1450.

Himeda, Y., Onozawa-Komatsuzaki, N., Sugihara, H., Arakawa, H., Kasuga, K. (2003). J. Mol Catal A Chem., 195 (1), 95-100.

Hong, Z., Cameron C.E. (2002). Pleiotropic mechanisms of ribavirin antiviral activities Progress in Drug Research. Springer, pp. 4169.

Ingold, C.K.(1969). Structure and Mechanism in Organic Chemistry, 2nd Edition.

Jamróz, M.H. (2004). Vibrational Energy Distribution Analysis. VEDA 4 program, Warsaw.

Jia, Y., Li, J. (2014). Chem. Rev., 115 (3), 1597-1621.

Kanamitsu, S.-i., Ito, K., Green, C.E., Tyson, C.A., Shimada, N., Sugiyama, Y. (2000). Prediction of in vivo interaction between triazolam and erythromycin based on in vitro studies using human liver microsomes and recombinant human CYP3A4 Pharm. Res., 17, pp. 419-426.

Karunakaran, V., Balachandran, V. (2012). Spectrochim. Acta A, 98, 229-239.

Krzysztof, S., Tomasz, T., Jolanta, R., Kazimierz, P., Martyna, K. (2008). Synthesis, determination of the lipophilicity, anticancer and antimicrobial properties of some fused 1,2,4-triazole derivatives. Eur. J. Med. Chem., 43, 404-419.

Kotan, G., Yüksek, H. (2016). Theoretical and Spectroscopic Studies of (E)-3-Benzyl-4-((4-Isopropylbenzylidene)-Amino)-1(Morpholinomethyl)-1H-1.2.4-triazol-5(4H)-one Molecule, JOTCSA, 3(3), 381-392.

Layer, R.W. (1963). Chem. Rev. 63, 489-510.

Lee, C., Yang, W., Parr, R.G., (1988). Development of the Colle-Salvetti correlation-energy formula into a functional of the electron density. Physical Review, 37, 785-789.

Lobo, B.L., Greene, W.L. (1997). Zolpidem: distinct from triazolam Ann. Pharmacother, 31 pp. 625-632

Mari, S.K., Bantwal, S.H., Nalilu, S.K. (2008). Eur. J. Med. Chem. 43, 309-314.S.

Merrick, J.P., Moran, D., Radom, L. (2007). An Evaluation of Harmonic Vibrational Frequency Scale Factors. Journal of Physical Chemistry, 111(45), 11683-11700.

Patel, R.V., Park. S.W. (2014). Eur. J. Med. Chem. 71, 24-30.

Perdew, J. P. (1986a). Density-functional approximation for the correlation energy of the inhomogeneous electron gas. Physical Review B, 33, 8822. (b) Perdew, J.P. (1986b). Physical Review B, 34, 7406.

Perdew, J.P. and Wang, Y. (1992). Accurate and simple analytic representation of the electron-gas correlation energy. Physical Review B, 45, 13244.

Pokharia, M., Yadav, S.K., Mishra, H., Pandey, N., Tilak, R., Pokharia, S. (2017). J. Mol.Struct., 1144, 324-337.

Postovskii, I.Y. \& Vereshchagina N.N. (1954). Z. Obshch Khim, 229, 2139.

Prakash, A., Adhikari, D. (2011). Application of Schiff bases and their metal complexes-A review. Int. J. Chem. Tech. Res. 3 (4), 1891-1896.

Rockstroh, J.K., Mudar, M., Lichterfeld, M., Nischalke, H.D., Klausen, G., Gölz, J., Dupke, S., Notheis, G., Stein, L., Mauss, S. (2002). Pilot study of interferon alpha high-dose induction therapy in combination with ribavirin for chronic hepatitis C in HIVco-infected patients AIDS, 16, pp. 2083-2085. 
Sheikhshoaie, I., Sharif, M.A. (2006). Acta Crystallogr. E 62, 3563-3565.

Suvitha, A., Periandy, S., Boomadevi, S., Govindarajan, M. (2014). Spectrochim. Acta A, 117, 216-224.

Tozkoparan, B., Küpeli, E.,Yesilada, E., Ertan, M. (2007). Preparation of 5-aryl-3-alkylthio-1,2,4-triazoles and corresponding sulfones with antiinflammatorye analgesic activity. Bioorg Med Chem., 15, 1808-1814.

Wellington, K., Faulds, D.M. (2002). Anastrozole Drugs., 62 pp, 2483-2490.

Wolinski, K., .Hilton, J.F., Pulay, P.J. (1990). Efficient implementation of the gauge-independent atomic orbital method for NMR chemical shift calculations. Journal of the American Chemical Society, 112, 512.

Vogel, G.W., Morris, D. (1992). The effects of estazolam on sleep performance and memory: a long-term sleep laboratory study of elderly insomniacs. J. Clin. Pharmacol, 32 pp, 647-651.

Yüksek, H., Gursoy-Kol, Ö., Kemer, G., Ocak, Z., Anıl, B. (2011). Synthesis and in-vitro antioxidant evaluation of some novel 4-(4substituted)benzylidenamino-4,5-dihydro-1H-1,2,4-triazol-5-ones. Indian J. Heterocy. Chemistry, 20, 325-330.

Yüksek, H., Kotan, G., Medetalibeyoğlu, H., Gürbüz, A., Alkan M. (2017). B3LYP ve HF Temel Setleri Kullanılarak Bazı 3-Alkil4-(2-asetoksi-3-metoksibenzilidenamino)-4,5-dihidro-1H-1.2.4-triazol-5-on Bileşiklerinin Deneysel ve Teorik Özelliklerinin İncelenmesi. CBÜ Fen Bil. Dergi, Cilt 13, Sayı 1, 193-204. 